\title{
Analytical model for estimation of eddy current and power loss in conducting plate and its application
}

\author{
Gautam Sinha and S. S. Prabhu \\ AMTD, Raja Ramanna Centre for Advanced Technology, Indore-452013, India
}

(Received 14 March 2011; published 1 June 2011)

\begin{abstract}
A model is developed to study the eddy current induced in a thin conducting but nonmagnetic plate of finite size when exposed to a time varying magnetic field. The applied field may be uniform or vary in space. This model can accurately estimate the eddy current contour in the plate and loss due to eddy current. Power losses for plates of various dimensions and at different frequencies are calculated to establish the accuracy of the model. We have also calculated the magnetic field generated by the induced eddy current when the plate of finite size is placed between the two parallel poles of a dipole magnet made of magnetic material of very high permeability. The force acting on the plate due to the interaction of the induced eddy current and the applied external field is also calculated. The model can predict the time variation of force and eddy current. The model may be applicable to understand the effect of eddy current on the vacuum chamber of an accelerator. Various other applications, where this model is useful, are also reported. The results are compared against the results obtained by a simulation using a finite element based code. Here the rectangular plate is considered but the model can be applicable for other geometries as well.
\end{abstract}

DOI: 10.1103/PhysRevSTAB.14.062401

PACS numbers: 41.20.Gz, 81.70.Ex

\section{INTRODUCTION}

The understanding of the nature of the eddy current induced in a metallic plate by the time dependent magnetic field and its consequences play a significant role in a wide range of technical and industrial applications. The eddy current in vacuum chambers used in rapid cycling synchrotrons, cores of fast cycling magnets, and accelerator cavities causes adverse effects which are already reported $[1,2]$. However, the heat generated by the eddy current is utilized in various applications like induction furnace, heat treating, melting, hardening, brazing, etc. [3-6]. The estimation of the eddy current is also used for nondestructive testing of conducting materials. A synchrotron accelerator or beam transport line contains various types of magnets to deflect and focus the beam, where the vacuum chambers are placed between the magnet poles. The eddy currents modify the $B$ field, which is generated by the accelerator magnets, for example dipoles and quadrupoles, so that the beam inside the accelerator beam pipe sees a different $B$ field than expected. For the beam this means the optics (or the lattice) of the accelerator is affected. In a next step, one might calculate the effect of the modified optics on the beam dynamics. To avoid the ill effect of eddy current in some accelerators, ceramic chambers are also used but they are difficult to make and it increases the cost of the machine. Prior knowledge of heat loss in the chambers may

Published by the American Physical Society under the terms of the Creative Commons Attribution 3.0 License. Further distribution of this work must maintain attribution to the author(s) and the published article's title, journal citation, and DOI. help in taking the decision judiciously. Several efforts are made to understand the field produced by the eddy current for different shapes and geometries of the chambers [7-9]. Because of the complex nature of the problem, an analytical solution may not be easy. Often numerical methods are adopted for solution of such problems [10,11]. There are many limitations and inconveniences associated with numerical methods. It takes a longer time and a considerable amount of CPU resources. Results will depend on mesh size and various other parameters, like material property, boundary conditions, linear or quadratic elements, etc. Even in the case of a small perturbation, the whole problem has to be solved. Therefore, an analytical solution is very important which helps in understanding the physical behavior of the electromagnetic field better. In spite of extensive studies of the eddy current in various areas regardless of applications, some areas remain unexplored [12-16], e.g., the eddy current and the force on a chamber when exposed to a nonuniform time varying magnetic field. Power loss predicted by earlier work was not very accurate [3]. In some cases, it was assumed that the eddy current varies linearly in space and the length of the chamber was large [2]. These cases motivated us to do the detailed calculation of the eddy current contour, power loss, and the field produced by it on a finite size conducting plate exposed to a uniform as well as a nonuniform time varying magnetic field.

In this paper we have proposed a complete model to understand the eddy current behavior on a rectangular plate and the consequences of it. We describe the problem mathematically by Maxwell's equations and obtain a partial differential equation. The problem is then converted to 
an inhomogeneous Helmholtz equation which has been solved with proper boundary conditions. It is assumed that the conducting plate is nonmagnetic and its thickness is small compared to the skin depth of the material. This means that we are concentrating on low frequency and thin plates. Both of these approximations are valid for accelerator vacuum chambers. The vacuum chambers are placed between magnet poles where the field is mostly perpendicular. So, the eddy current induced by the normal component of the applied magnetic field is considered here and the tangential component is neglected. We have considered two different cases: (i) case I-the plate is exposed to a uniform time varying field; and (ii) case II-the field is uniform on some portion of the plate but exponentially reducing with distance to the edge of the plate. Case I is applicable to chambers of any booster or rapid cycling machine. Case II is applicable to the chambers of a storage ring where electrons are injected at lower energy and then ramped up to the nominal energy [17]. We have also calculated the magnetic field generated by the induced eddy current when a plate of finite size is placed between the two parallel poles of a dipole magnet made of magnetic material of very high permeability. The only assumption that we have made is that the permeability is very high so that the boundary condition for the field at the interface between the air and the iron poles can easily be achieved by using the image current method. The present model can provide accurately the following parameters: (i) eddy current contour in the plate; (ii) power loss due to the eddy current in the plate; (iii) force acting on it; and (iv) magnetic field produced by the induced eddy current.

For quick verification of the analytical model, the results are compared against those obtained by simulation using a finite element (FEM) based code, like OPERA [10]. These calculations will be useful in understanding the effect of the eddy current in the vacuum chambers, used in particle accelerators, subjected to a time varying field. The chambers of a synchrotron radiation storage ring are exposed to a nonuniform magnetic field. In that case the study of the adverse effect of eddy current, which is not explored before, is the main thrust of the present work.

\section{THEORETICAL MODEL}

Consider that a conducting nonmagnetic plate of width $a$, length $b$, and depth $d(d \ll a$ and $b$ ) is exposed to a time varying magnetic field directed along the vertical $(Z)$ direction (see Fig. 1). The nature of the field variation is assumed to be harmonic:

$$
B(x, y, t)=B_{0}(x, y) \cos (\omega t) \hat{z} .
$$

We consider the following spatial variations of $B_{0}(x, y)$ :

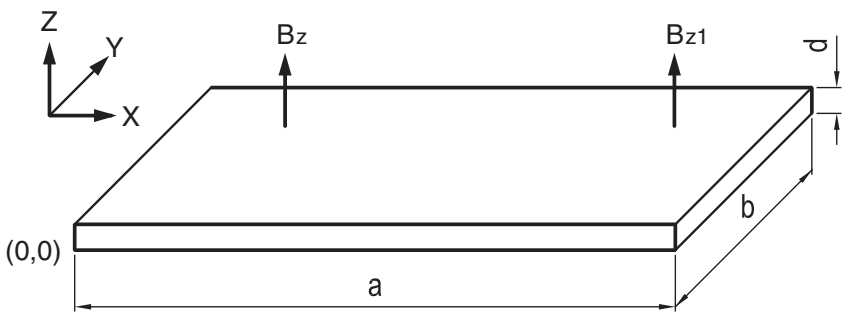

FIG. 1. Coordinate system, plate geometry, and the applied field. For case I, the field $B_{z}=B_{z 1}$ is uniform throughout the plate. For case II, $B_{z}$ is uniform up to a distance, $c(c<a)$ and then exponentially decreasing with distance, $B_{z 1}=f(x)$.

case I: $B_{0}(x, y)=B_{0}$ for $0 \leq x \leq a, 0 \leq y \leq b$

case II: $B_{0}(x, y)=B_{0}$ for $0 \leq x \leq c, 0 \leq y \leq b$

$$
=B_{0} e^{-(x-c) / \lambda} \text { for } c \leq x \leq a, 0 \leq y \leq b,
$$

where $c<a$. Hereafter we will refer to these as case I and case II, respectively. The Maxwell's equations in the absence of the plate can be written as

$$
\vec{\nabla} \times \vec{B}=\mu_{0} \varepsilon_{0} \frac{\partial \vec{E}}{\partial t} \quad \vec{\nabla} \times \vec{E}=-\frac{\partial \vec{B}}{\partial t},
$$

where $\vec{E}$ is the electric field, $\mu_{0}$ is the permeability, and $\varepsilon_{0}$ is the dielectric constant of free space. The time varying magnetic field will induce an eddy current in the conducting plate. The eddy current will produce induced field $\vec{H}_{i}$ that will oppose the applied field. So, the Maxwell's equations in the presence of the plate can be written as

$$
\begin{aligned}
\vec{\nabla} \times\left(\vec{B}+\mu_{0} \vec{H}_{i}\right) & =\mu_{0} \vec{J}+\mu_{0} \varepsilon_{0} \frac{\partial \vec{E}}{\partial t} \\
\vec{\nabla} \times \vec{E} & =-\frac{\partial\left(\vec{B}+\mu_{0} \vec{H}_{i}\right)}{\partial t},
\end{aligned}
$$

where $\vec{J}$ is the current density in the plate. Using Eqs. (3) and (4) we find

$$
\vec{\nabla} \times \vec{H}_{i}=\vec{J} .
$$

According to the Lenz law, the induced emf and hence the eddy current will be of the form $\sin (\omega t)$ as the applied field is of the form $\cos (\omega t)$ [Eq. (1)]. In the low frequency regime, the induced field may be expressed as

$$
\vec{E}=\vec{E}_{0} \sin (\omega t) \quad \vec{H}_{i}=\vec{H}_{0} \sin (\omega t) .
$$

Eddy current flows in a close loop, in other words, $\vec{\nabla} \cdot \vec{J}=0$. Therefore, $\vec{J}$ can be written as the curl of a potential function $u(x, y)$ :

$$
\vec{J}=\frac{1}{d} \vec{\nabla} \times\{u(x, y) \hat{z}\} \sin (\omega t) .
$$

Using Eqs. (5) and (7) we obtain 


$$
\begin{aligned}
\vec{\nabla}_{i} \times\left(\vec{H}_{i}-\frac{u(x, y)}{d} \hat{z} \sin (\omega t)\right)= & 0 \\
\operatorname{so}\left(\vec{H}_{i}-\frac{u(x, y)}{d} \hat{z} \sin (\omega t)\right)= & \vec{\nabla} V(x, y, z) \sin (\omega t) \\
\vec{H}_{i}= & \left(\frac{u(x, y)}{d} \hat{z}+\vec{\nabla} V(x, y, z)\right) \\
& \times \sin (\omega t),
\end{aligned}
$$

where $V(x, y, z)$ is a scalar magnetic potential. In the present model the eddy current is flowing on the surface of the plate, i.e., $j_{z}=0$. We are interested in finding the eddy current distribution on the plate. The $Z$ component of the field will depend on $\partial V(x, y, z) / \partial z$ which should be a function of $x$ and $y$ on the surface of the plate. Therefore, we have presumed that $\partial V(x, y, z) / \partial z$ will be related to a general function $u(x, y)$, already assumed. So, without loss of generality, we assumed the induced field along the $Z$ direction to be

$$
H_{i}^{z}=\left(\frac{u(x, y)}{d}+g u(x, y)\right) \sin (\omega t)
$$

where $g$ is constant and $H_{i}^{z}$ is evaluated on the surface of the metallic plate. Using Ohm's law $\vec{J}=\sigma \vec{E}$, we find from Eq. (4)

$$
\frac{\partial J_{y}}{\partial x}-\frac{\partial J_{x}}{\partial y}=\sigma\left\{B_{0}(x, y) \omega \sin (\omega t)-\mu_{0} H_{0} \omega \cos (\omega t)\right\} .
$$

Substituting expressions from Eqs. (7) and (9) to Eq. (10), we obtain

$$
\begin{aligned}
\left(\frac{\partial^{2}}{\partial x^{2}}+\frac{\partial^{2}}{\partial y^{2}}\right) u(x, y) \sin (\omega t)-k^{2}(1+g d) \cos (\omega t) u(x, y) \\
=-\frac{d}{\mu_{0}} k^{2} B_{0}(x, y) \sin (\omega t)
\end{aligned}
$$

where $k^{2}=\mu_{0} \sigma \omega$ and $\sigma$ is the conductivity of the plate. The above inhomogeneous Helmholtz equation can be solved with certain boundary conditions to obtain $u(x, y)$ and hence the eddy current contour in the plate.

\section{A. EDDY CURRENT}

We now solve Eq. (11) in two specific cases as given in Eq. (2). The relevant boundary condition is given by

$$
u(0, y)=u(a, y)=u(x, 0)=u(x, b)=0 .
$$

The eigenfunction $u_{n m}(x, y)$ of the equation

$$
\left(\frac{\partial^{2}}{\partial x^{2}}+\frac{\partial^{2}}{\partial y^{2}}\right) u_{n m}(x, y)=\lambda_{n m} u_{n m}(x, y)
$$

with the above boundary condition can then be written as

$$
u_{n m}(x, y)=N_{n m} \sin \left(\nu_{n} x\right) \sin \left(\mu_{m} y\right)
$$

where $\nu_{n}=\frac{n \pi}{a}, \quad \mu_{m}=\frac{m \pi}{b}$ ( $n, m$ are integers), and $N_{n m}=\frac{2}{\sqrt{a b}}$.

The general solutions $u^{\mathrm{I}}(x, y)$ and $u^{\mathrm{II}}(x, y)$ of Eq. (11) for two cases as mentioned in Eq. (2) may then be expressed as

$$
\begin{aligned}
& u^{\mathrm{I}}(x, y)=\sum_{n, m=1}^{\infty} A_{n m}^{\mathrm{I}} N_{n m} \sin \left(\nu_{n} x\right) \sin \left(\mu_{m} y\right) \\
& u^{\mathrm{II}}(x, y)=\sum_{n, m=1}^{\infty} A_{n m}^{\mathrm{II}} N_{n m} \sin \left(\nu_{n} x\right) \sin \left(\mu_{m} y\right),
\end{aligned}
$$

where

$$
A_{n m}^{\mathrm{I}}=\frac{2 k^{2} B_{0} d}{\mu_{0} \sqrt{a b}} \frac{K_{n m}^{0}(a, b) \sin (\omega t)}{\left(\nu_{n}^{2}+\mu_{m}^{2}\right) \sin (\omega t)+k^{2}(1+g d) \cos (\omega t)}
$$

and $A_{n m}^{\mathrm{II}}=\frac{2 k^{2} B_{0} d}{\mu_{0} \sqrt{a b}} \frac{K_{n m}(a, b, c) \sin (\omega t)}{\left(\nu_{n}^{2}+\mu_{m}^{2}\right) \sin (\omega t)+k^{2}(1+g d) \cos (\omega t)}$

with

$$
\begin{aligned}
K_{n m}^{0}(a, b)= & \frac{\left\{1-\cos \left(\nu_{n} a\right)\right\}\left\{1-\cos \left(\mu_{m} b\right)\right\}}{\nu_{n} \mu_{m}} \\
K_{n m}(a, b, c)= & \left(1-\frac{\lambda^{2} \nu_{n}^{2}}{1+\lambda^{2} \nu_{n}^{2}}\right) K_{n m}^{0}(c, b) \\
& +\left(\frac{\lambda^{2} \nu_{n}^{2}}{1+\lambda^{2} \nu_{n}^{2}}\right) e^{(c-a) / \lambda} K_{n m}^{0}(a, b) \\
& +\left(\frac{\lambda^{2} \nu_{n}^{2}}{1+\lambda^{2} \nu_{n}^{2}}\right) L_{n m}^{0}(c, b) \\
& -\left(\frac{\lambda^{2} \nu_{n}^{2}}{1+\lambda^{2} \nu_{n}^{2}}\right) e^{(c-a) / \lambda} L_{n m}^{0}(a, b) \quad \text { and } \\
L_{n m}^{0}(a, b)= & \frac{\left\{1-\cos \left(\nu_{n} a\right)\right\}}{\nu_{n} \mu_{m}}\left\{1+\frac{\sin \left(\nu_{n} a\right)}{\lambda \nu_{n}}\right\}
\end{aligned}
$$

are obtained using the orthogonality condition $\int_{0}^{a} \partial x \int_{0}^{b} \partial y u_{n m}(x, y) u_{n^{\prime} m^{\prime}}(x, y)=\delta_{n n^{\prime}} \delta_{m m^{\prime}}$. Therefore, the eddy current density and the induced field due to the eddy current on the surface of the plate can be expressed as 


$$
\begin{aligned}
j_{x} & =\frac{1}{d} \frac{\partial u(x, y)}{\partial y} \sin (\omega t)=\sum_{n, m=1}^{\infty} \frac{\mu_{m}}{d} A_{n m}^{\alpha} N_{n m} \sin \left(\nu_{n} x\right) \cos \left(\mu_{m} y\right) \sin (\omega t) \\
j_{y} & =-\frac{1}{d} \frac{\partial u(x, y)}{\partial x} \sin (\omega t)=-\sum_{n, m=1}^{\infty} \frac{\nu_{n}}{d} A_{n m}^{\alpha} N_{n m} \cos \left(\nu_{n} x\right) \sin \left(\mu_{m} y\right) \sin (\omega t) \\
H_{i}^{z} & =\frac{1}{d}(1+g d) u(x, y) \sin (\omega t)=\sum_{n, m=1}^{\infty} \frac{1}{d}(1+g d) A_{n m}^{\alpha} N_{n m} \sin \left(\nu_{n} x\right) \sin \left(\mu_{m} y\right) \sin (\omega t),
\end{aligned}
$$

where $\alpha$ represents I or II.

The value of the constant $g$ needs to be determined selfconsistently. This has been performed as follows. Since the eddy current is maximum at $\omega t=\frac{\pi}{2}$, we first evaluate $j_{x}$ and $j_{y}$ using Eqs. (16) and (17). Note that at $\omega t=\frac{\pi}{2}$, the coefficient $A_{n m}$ of Eq. (16) is independent of $g$ because $(1+g d) \cos (\omega t)$ is zero. Therefore, the exact values of $j_{x}$ and $j_{y}$ are obtained even without knowing the value of $g$. Then $H_{i}^{z}$ is evaluated on the surface of the plate using Eq. (17) and is proportional to $(1+g d)$. By knowing the values of $j_{x}$ and $j_{y}$, the $B$ field on the surface of the plate can also be evaluated using Biot-Savart's law (see Sec. II D). The value of $(1+g d)$ is obtained by comparing the field values obtained using two different methods as explained above. Finally, the value of $g$ is obtained by using the value of $d$ which is the thickness of the plate. The value of $g$ depends on whether the plate is placed between two parallel poles of a dipole magnet or the gap between the poles, and the permeability of the material of the magnet poles. While evaluating Eq. (17) we have taken the value of $m$ and $n$ up to 300 because beyond this limit the change in $j$ is less than $0.001 \%$ (see Fig. 3).

\section{B. POWER LOSS}

The calculation of power loss plays a very significant role in various applications. Therefore, the real test of the present model depends on the accuracy of the loss prediction. Power loss $P$ can be expressed using the well-known volume integral

$$
P=\frac{1}{\sigma} \int_{V} J^{2} \partial V=\frac{1}{\sigma} \int_{V}\left(j_{x}^{2}+j_{y}^{2}\right) \partial V .
$$

Substituting the expressions of $j_{x}$ and $j_{y}$ from Eq. (17) to Eq. (18) and performing the integrals, we find the final expression of the power loss as

$$
P=\frac{1}{\sigma d} \sum_{n, m=1}^{\infty} A_{n m}^{\alpha} A_{n m}^{\alpha}\left(\nu_{n}^{2}+\mu_{m}^{2}\right) \sin ^{2}(\omega t)
$$

The power loss for case I and case II can be obtained from Eq. (19) by putting the values of the coefficient $A_{n m}^{\mathrm{I}}$ or $A_{n m}^{\mathrm{II}}$ from Eq. (16). Note that $A_{n m}^{\alpha}$ depends on $\omega t$. The average power loss $\langle P\rangle$ is the average of $P$ over a cycle.

\section{MAGNETIC FORCE}

If an eddy current flows through the conducting plate then it will experience a force in the presence of the magnetic field. The $B$ field is uniform throughout the plate. The amplitude of the eddy current increases symmetrically as we move away from the center of the plate. But, the sign of the eddy current $j_{y}\left(j_{x}\right)$ is positive on the right (lower) side of the plate and negative on the left (upper) side (Figs. 4 and 5). So, the resulting force on one half of the plate will be balanced by the other half and there will be no resultant force acting on the plate (case I). However, this balance will be disturbed when the plate is exposed to a nonuniform field. It is very important for the stability of any system to estimate the magnitude of the force. The force can be expressed as

$$
\vec{F}=\int_{V}(\vec{J} \times \vec{B}) \partial V .
$$

In case II, there will be a net force acting on the plate. Substituting $\vec{J}$ from Eq. (17) to Eq. (20), the net force acting along the $x$ direction can be written as

$$
\begin{aligned}
\vec{F}_{X}= & B_{0} \hat{x} \cos (\omega t) \sin (\omega t) \sum_{n, m=1}^{\infty} \frac{1}{\mu_{m}} A_{n m}^{I I} N_{n m} \\
& \times\left[1-\cos \left(\mu_{m} b\right)\right]\left(\sin \left(\nu_{n} c\right)+\left(\frac{\lambda^{2} \nu_{n}^{2}}{1+\lambda^{2} \nu_{n}^{2}}\right)\right. \\
& \times\left[e^{(c-a) / \lambda}\left\{\sin \left(\nu_{n} a\right)-\frac{1}{\lambda \nu_{n}} \cos \left(\nu_{n} a\right)\right\}\right. \\
& \left.\left.-\left\{\sin \left(\nu_{n} c\right)-\frac{1}{\lambda \nu_{n}} \cos \left(\nu_{n} c\right)\right\}\right]\right) .
\end{aligned}
$$

Similarly, the force in the other direction can also be calculated. As the force depends on the eddy current we have taken the sum of $m$ and $n$ up to 300 only without losing too much accuracy.

\section{FIELD DUE TO EDDY CURRENT IN THE PLATE IN THE PRESENCE OF MAGNETIC POLES HAVING VERY HIGH PERMEABILITY}

We have already calculated the eddy current distribution in the conducting plate induced by time varying magnetic field. We have assumed that the thickness of the plate is uniform and quite small compared to the skin depth. This assumption may be fine for the vacuum chambers used in 
accelerators which produce synchrotron radiation. The source current density for which the induced field needs to be calculated is lying on the $x-y$ plane. The magnetic field at a point $P(x, y, z)$ due to a source current at $\left(x^{\prime}, y^{\prime}, z^{\prime}\right)$ can be expressed using the Biot-Savart law:

$$
B(x, y, z)=\frac{\mu_{0}}{4 \pi} \int_{V} \frac{j \times\left(r-r^{\prime}\right)}{\left(r-r^{\prime}\right)^{3}} \partial x^{\prime} \partial y^{\prime} d
$$

where $r-r^{\prime}=\left(x-x^{\prime}, y-y^{\prime}, z-z^{\prime}\right)$.

By taking $z^{\prime}=0$ and taking the curl we get

$$
\begin{aligned}
& B_{0}(x, y, z) \\
& =\frac{d \mu_{0}}{4 \pi} \int_{0}^{a} \partial x^{\prime} \int_{0}^{b} \partial y^{\prime}\left[\begin{array}{c}
\frac{j_{x}\left(y-y^{\prime}\right)-j_{y}\left(x-x^{\prime}\right)}{\left\{\left(x-x^{\prime}\right)^{2}+\left(y-y^{\prime}\right)^{2}+z^{2}\right\}^{3 / 2}} \hat{z}+ \\
\frac{\left(-j_{x} z\right)}{\left\{\left(x-x^{\prime}\right)^{2}+\left(y-y^{\prime}\right)^{2}+z^{2}\right\}^{3 / 2}} \hat{y}+ \\
\frac{j_{y} z}{\left\{\left(x-x^{\prime}\right)^{2}+\left(y-y^{\prime}\right)^{2}+z^{2}\right\}^{3 / 2}} \hat{x}
\end{array}\right] .
\end{aligned}
$$

The above expression is true in the absence of any magnetic material. The eddy current is distributed in the plate. We consider a small strip of the plate as a current carrying conductor. Therefore, we will now calculate the field when the current carrying conductors are located between the two parallel iron poles with very high permeability. Then the boundary condition for the field at the interface between the air and the iron poles can easily be achieved by using the image current method [2]. Because of these two parallel poles, the image currents of the source current density $j$ at $\left(x^{\prime}, y^{\prime}, z^{\prime}\right)$ will be located at $z^{\prime}=$ $0, f, 2 f, \ldots$, and so on. Here $f$ is the distance between these two iron poles. Equation (23) represents the field for the $z^{\prime}=0$ condition. Therefore, the field in the $z$ direction in the presence of magnetic poles can be expressed by adding terms arising from image currents to Eq. (23):

$$
\vec{B}(x, y, z)=\sum_{n=-\infty}^{\infty} \vec{B}_{n}(x, y, z) \text { where } \vec{B}_{n}(x, y, z) \approx \frac{d \mu_{0}}{4 \pi} \int_{0}^{a} \partial x^{\prime} \int_{0}^{b} \partial y^{\prime}\left[\frac{j_{x}\left(y-y^{\prime}\right)-j_{y}\left(x-x^{\prime}\right)}{\left\{\left(x-x^{\prime}\right)^{2}+\left(y-y^{\prime}\right)^{2}+(z-n f)^{2}\right\}^{3 / 2}} \hat{z}\right] .
$$

Here, we have assumed the value of $\mu$ very large but, in practical cases, it will have finite values. Therefore, the field calculated by using Eq. (24) may be marginally higher than the actual field. Here the sum up to 10 is sufficient as the change of the field value for $n>10$ is less than $0.001 \%$.

\section{E. SIMULATION USING A FEM CODE}

The OPERA-3D [10] analysis program ELEKTRA can be used to compute time varying electromagnetic fields in three dimensions including the effects of eddy currents. The program incorporates state of the art algorithms for the calculation of electromagnetic fields and advanced finite element numerical analysis procedures. ELEKTRA/SS calculates steady-state ac currents (the time harmonic form) where all fields and potentials are oscillating at the same angular frequency $\omega$. So, $A(t)=A_{c} e^{i \omega t}$ and $U(t)=$ $U_{c} e^{i \omega t}$. Time varying magnetic $(B)$ and electric $(E)$ fields can be expressed in terms of vector $(A)$ and scalar $(U)$ potential as $B=\nabla \times A$ and $E=-\frac{\partial A}{\partial t}-\nabla U$. Using the relations $\nabla \times H=j=\sigma E$ and $\nabla . j=0$, we have

$$
\begin{aligned}
\nabla \times \frac{1}{\mu} \nabla \times A & =-\sigma \frac{\partial A}{\partial t}-\sigma \nabla U \\
\nabla \cdot(\sigma \nabla U)+\nabla \cdot\left(\sigma \frac{\partial A}{\partial t}\right) & =0 .
\end{aligned}
$$

Using the time dependent part we get

$$
\begin{aligned}
\nabla \times \frac{1}{\mu} \nabla \times A_{c}+i \omega \sigma A_{c}+\sigma \nabla U_{c} & =0 \\
\nabla \cdot\left(\sigma \nabla U_{c}\right)+i \omega \sigma \nabla A_{c} & =0 .
\end{aligned}
$$

OPERA-3D used the finite element method to obtain solutions of the partial differential equations with proper boundary conditions. From the solution, the values of $A$ and $U$ can be obtained that will provide $B, E$, and $j$. Partial differential and integral equations describe the spatial and temporal variation of a field either directly in terms of the field variable, for example the magnetic flux density $\mathbf{B}$, or more often using a potential function that is related to the field by a gradient or a curl operation. The finite element method is generally applicable to any problem with any type of nonlinearity. The method is based on division of the domain of the equation (volume of space in which the equation is satisfied) into small volumes (the finite elements). Within each finite element a simple polynomial is used to approximate the solution.

This software is installed in HP DL 380 G5 server having xeon $3.73 \mathrm{GHz}$ dual core with 8GB RAM and Red Hat Enterprise Linux 4.0. To study case I, a dipole magnet is modeled and a metallic plate is placed between the poles. The dimensions of the plate are $0.1 \times 0.1 \mathrm{~m}^{2}$. The pole width and length of the magnet are taken as $0.2 \times 0.2 \mathrm{~m}^{2}$ so that it generates a uniform field on the plate. The mesh size of the plate is taken as $1 \times 1 \mathrm{~mm}^{2}$ in the $x-y$ plane and $0.5 \mathrm{~mm}$ along thickness $(z)$ whereas the mesh near the back leg of the magnet is $8 \times 8 \mathrm{~mm}^{2}$ in the $x-z$ plane. This model is solved with $1 / 4$ symmetry and the total nodes in the model are 2098791 . The accuracy of the field calculation is better than $0.1 \%$. It takes typically four hours to solve for one frequency. Time required will be more for better accuracy. To study case II, the dimensions of the plate are taken as $0.3 \times 0.1 \mathrm{~m}^{2}$ so that the field 
along the width varies with distance and the field along the length remains constant as explained in Eq. (2).

\section{RESULTS AND DISCUSSION}

We will first study the symmetric case, i.e., case I. Vacuum chambers are placed within the pole width of the dipole magnets in the rapid cycling machines and boosters. In these cases the chambers are exposed to the magnetic field produced by the magnet which is uniform (neglecting edge effect) and symmetric with respect to the center of the chambers. These calculations will be useful in understanding the eddy current behavior in such machines. To study the effect analytically we have simplified this and so a stainless steel (SS) plate is placed between two poles of a dipole magnet which creates a uniform $B$ field on the plate in the vertical direction. The dimensions of the plate are chosen in such a way as to avoid the edge effect in the case of simulation. The width $(a)$, length $(b)$, and thickness $(d)$ of the plate are $0.1,0.1$, and $0.002 \mathrm{~m}$, respectively. The model used for simulation is shown in Fig. 2. The pole width and the length of the magnet are 0.2 and $0.2 \mathrm{~m}$, respectively. The field uniformity in the plate is $0.08 \%$. The average peak field on the plate and the frequency of the field are $0.92321 \mathrm{~T}$ and $50 \mathrm{~Hz}$, respectively, and the conductivity of the plate is $1.33 \times 10^{6} \mathrm{~s} / \mathrm{m}$.

The values of the current density can be obtained analytically by using Eq. (17). The value of $(1+g d)$ is 0.048 for the present geometry. However, Eq. (17) contains a sum over an infinite series. The variation of the current density with the indices of the sum, $m$ and $n$, is plotted in Fig. 3 .

The value of $J_{y}$ is changing by $0.002 \%$ and $0.0006 \%$ if the index of sum changes from 200 to 300 and 300 to 400 , respectively. Therefore, we have taken index of sum $m=n$ as 300 throughout the paper for calculation of the current density.

The eddy current is symmetric about the midpoint of the plate in the presence of a uniformly distributed time

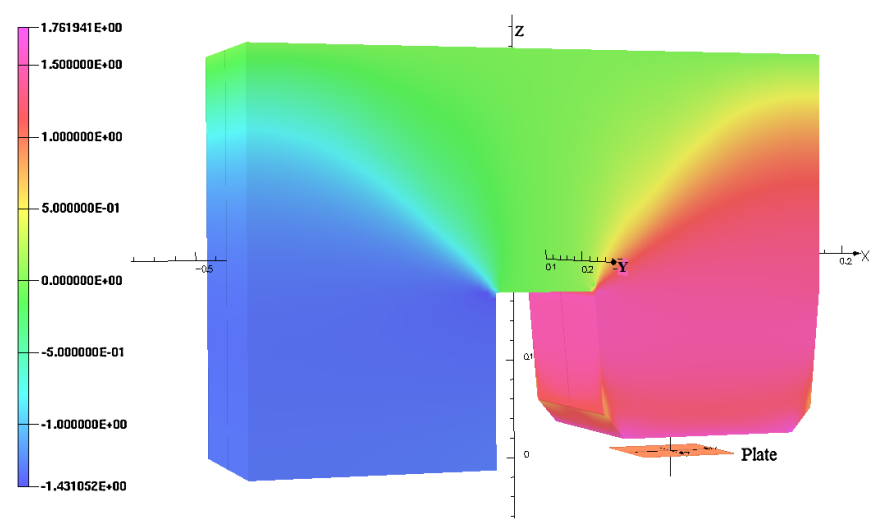

FIG. 2. The 3D model used for simulation study is shown here. Different colors represent the vertical component of the field $B_{z}$ (from $-1.431052 \mathrm{~T}$ to $1.761941 \mathrm{~T}$ ) in the magnet core and the plate.

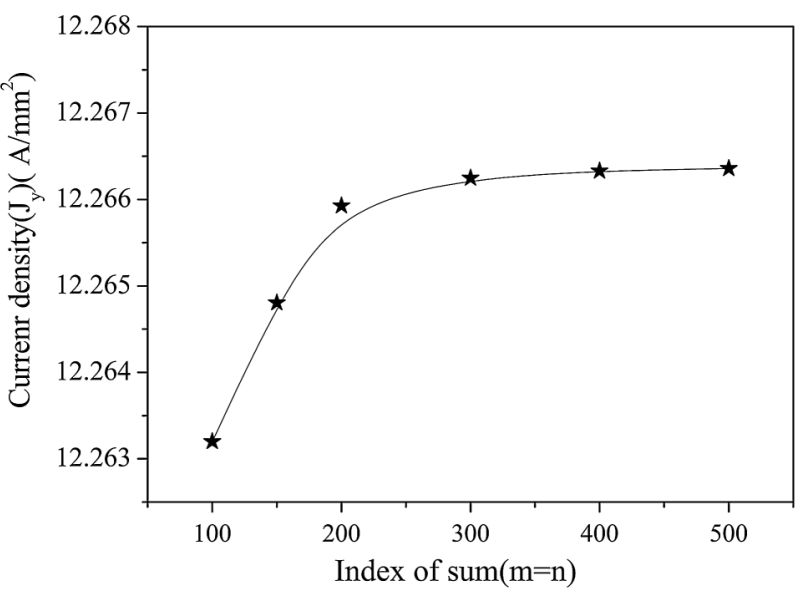

FIG. 3. Evaluation of the eddy current from analytical expressions for different indices of the sum $(m=n)$ in Eq. (17). The solid line is just a guide to the eye.

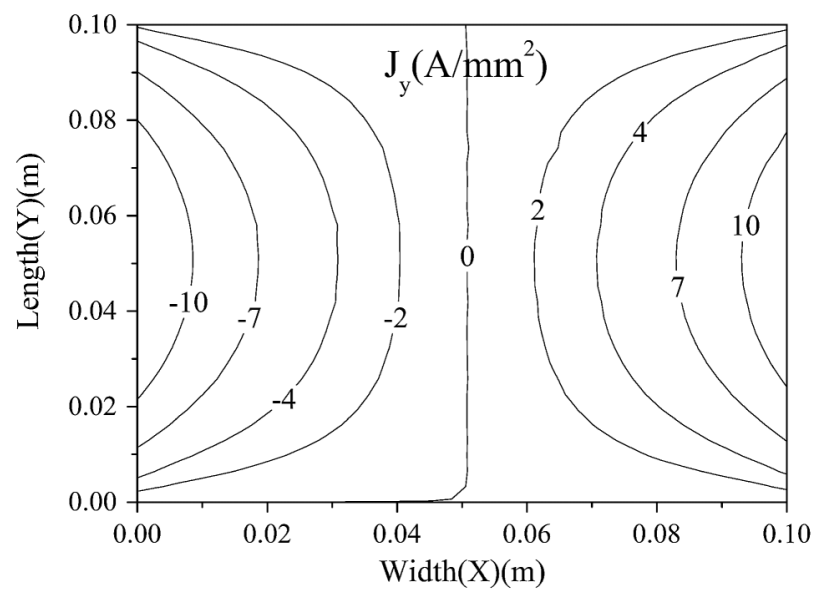

FIG. 4. Contour plot of $Y$ component of the eddy current density, $j_{y}$ in the SS plate of dimension $(0.1,0.1,0.002) \mathrm{m}^{3}$ for $50 \mathrm{~Hz}$ frequency and $0.92321 \mathrm{~T}$ peak field.

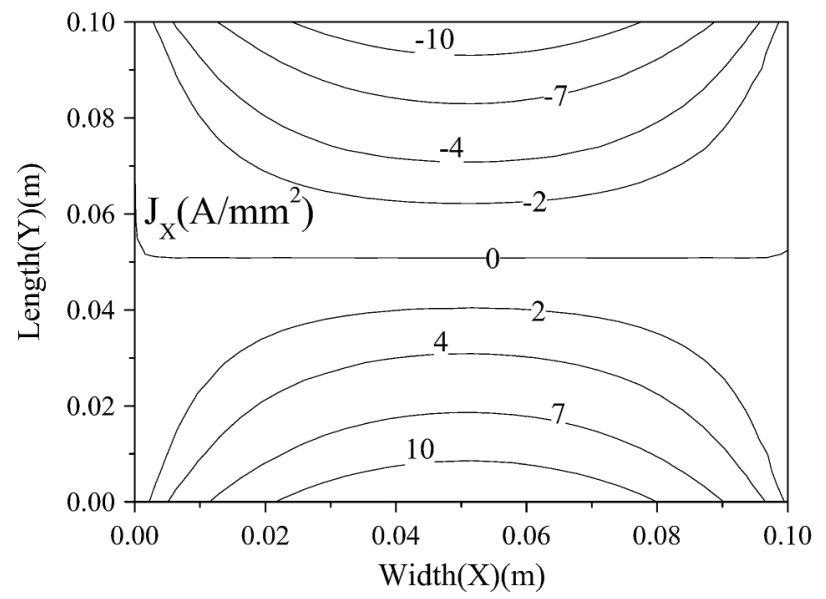

FIG. 5. Contour plot of $X$ component of the eddy current density, $j_{x}$ in the SS plate of dimension $(0.1,0.1,0.002) \mathrm{m}^{3}$ for $50 \mathrm{~Hz}$ frequency and $0.92321 \mathrm{~T}$ peak field. 


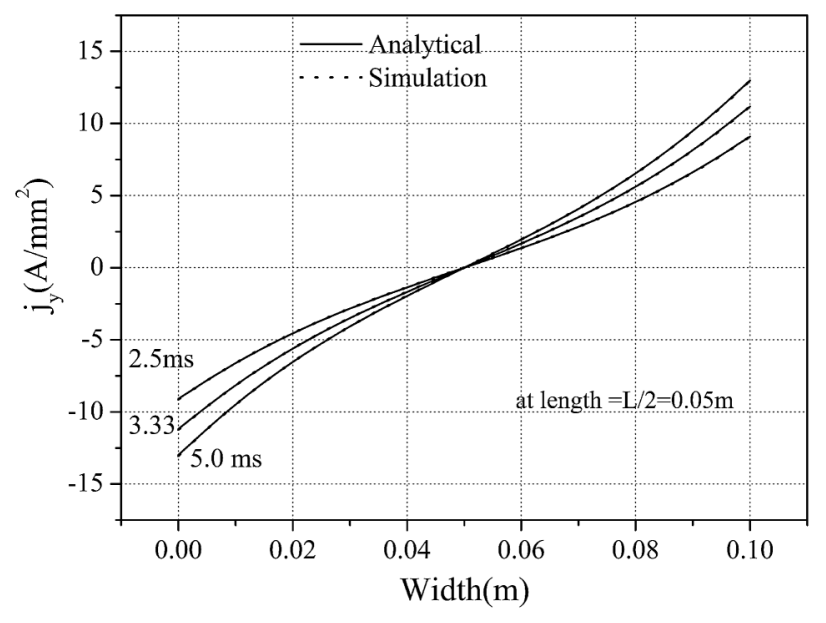

FIG. 6. Variation of the eddy current density, $j_{y}$ in the SS plate of dimension $(0.1,0.1,0.002) \mathrm{m}^{3}$ for $50 \mathrm{~Hz}$ frequency and $0.92321 \mathrm{~T}$ peak field at different times obtained from two different methods.

varying magnetic field. The $Y$ component of the eddy current $j_{y}$ in the plate is shown in Fig. 4. It is maximum at the center of the right edge as expected and it passes through zero at the line bisecting the plate along the width and the minimum is at the center of the left edge. Because of the square shape of the plate, the nature of the $X$ component of the eddy current $j_{x}$ will also be the same. Figure 5 shows the variation of $j_{x}$ in the plate for $50 \mathrm{~Hz}$ frequency.

The values of the current density, predicted by the analytical model, are in good agreement with the simulation values obtained using a 3D FEM code [10]. The values of the eddy current density obtained from two different methods are plotted in Fig. 6 for comparison. The agreements are within $0.15 \%$. This work may be extended for rectangular vacuum chambers with some approximations. A rectangular chamber is made of two such horizontal plates connected by two thin (few mm width) vertical plates at the end. Neglecting the contribution of the vertical plates, the eddy current in the chamber will be the same as that of two horizontal plates separated by a distance.

It is to be noted that the variation of the eddy current is not linear along the width as considered in earlier studies [2]. Their nature varies at different intermediate time intervals of a cycle. This is one of the new findings of the present work.

The power loss calculation, due to the flow of the eddy current, is also very important for various technical and engineering applications. The power loss predicted by the analytical model can be evaluated by using Eq. (19). It depends on the square of the eddy current. Here the sum over $m=n$ is taken from 1 to 300 . Losses are calculated at various frequencies and for different dimensions of the plates. The losses, averaged over a cycle, are given in Table I. For a quick verification of our results we have compared the power loss obtained from a 3D FEM code, OPERA 12.0. The analytical results are in good agreement with the results obtained from simulation. To compare our results with the simulation results, we have taken the average $B$ field on the plate rather than the field at the center of the plate.

The magnetic field produced by the eddy current is also evaluated. The presence of the two parallel iron poles enhanced the field value. We have estimated the field utilizing the image current expressed in Eq. (24). The $B_{Z}$ field generated due to the eddy current at $t=5 \mathrm{~ms}$ is plotted in Fig. 7 for $50 \mathrm{~Hz}$ frequency. In the central zone it shows that the field varies with the square of the distance, a typical sextupole like variation as expected. However, the nature and content of the multipole depends on the distance from the plate and the point of observation. The variations of the vertical field component for different distances from the surface of the plate are plotted in Fig. 8. The vertical component of the field obtained from the simulation is also plotted in the same graph for comparison. In our model we have taken the permeability of the material of the magnet poles to be very high but in the simulation some finite value was taken ( $B-H$ curve for a standard silicon steel is used). So, the estimated field value is slightly higher than the

TABLE I. Average power loss at different frequencies for stainless steel (SS) and aluminum (Al) plates of conductivity $1.33 \times 10^{6}$ and $16.95 \times 10^{6} \mathrm{~s} / \mathrm{m}$, respectively.

\begin{tabular}{|c|c|c|c|c|}
\hline $\mathrm{SS}(0.1,0.1,0.001) \mathrm{m}^{3}$ & $\mathrm{P}(\mathrm{W}) @ 5 \mathrm{~Hz}$ & $\mathrm{P}(\mathrm{W}) @ 10 \mathrm{~Hz}$ & $\mathrm{P}(\mathrm{W}) @ 25 \mathrm{~Hz}$ & $\mathrm{P}(\mathrm{W}) @ 50 \mathrm{~Hz}$ \\
\hline Our model & 2.270 & 9.08 & 56.76 & 226.97 \\
\hline Simulation & 2.266 & 9.06 & 56.62 & 226.60 \\
\hline \multicolumn{5}{|l|}{$\mathrm{SS}(0.05,0.1,0.001) \mathrm{m}^{3}$} \\
\hline Our model & 0.47 & 1.88 & 11.78 & 47.14 \\
\hline Simulation & 0.47 & 1.88 & 11.78 & 47.13 \\
\hline \multicolumn{5}{|l|}{$\mathrm{Al}(0.1,0.1,0.002) \mathrm{m}^{3}$} \\
\hline Our model & 50.06 & 199.94 & 1249.6 & 4998.5 \\
\hline Simulation & 49.99 & 199.83 & $\cdots$ & $\cdots$ \\
\hline
\end{tabular}




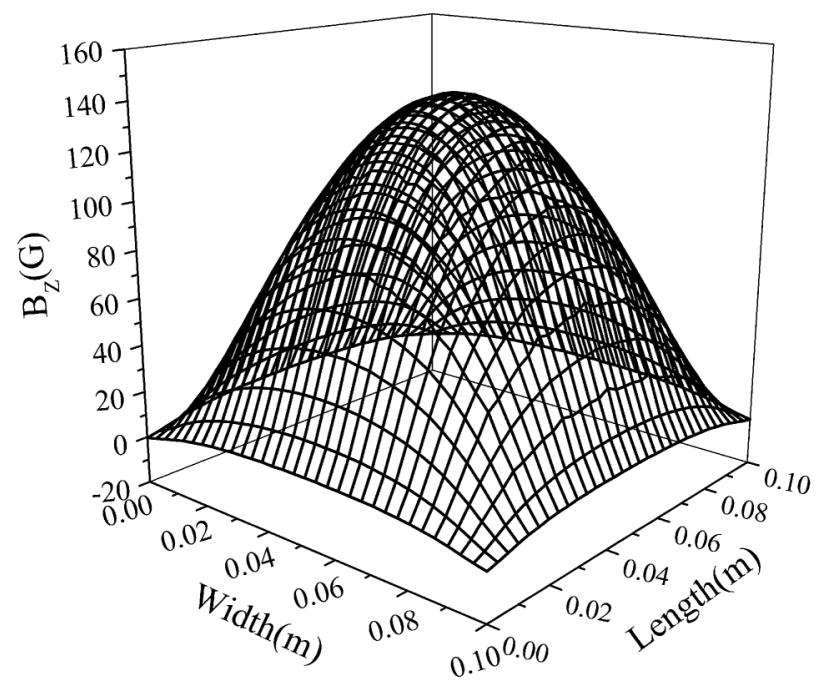

FIG. 7. 2D plot of the vertical component of the field due to the eddy current flow in the SS plate for $50 \mathrm{~Hz}$ frequency at a distance $z=10 \mathrm{~mm}$ from the plate.

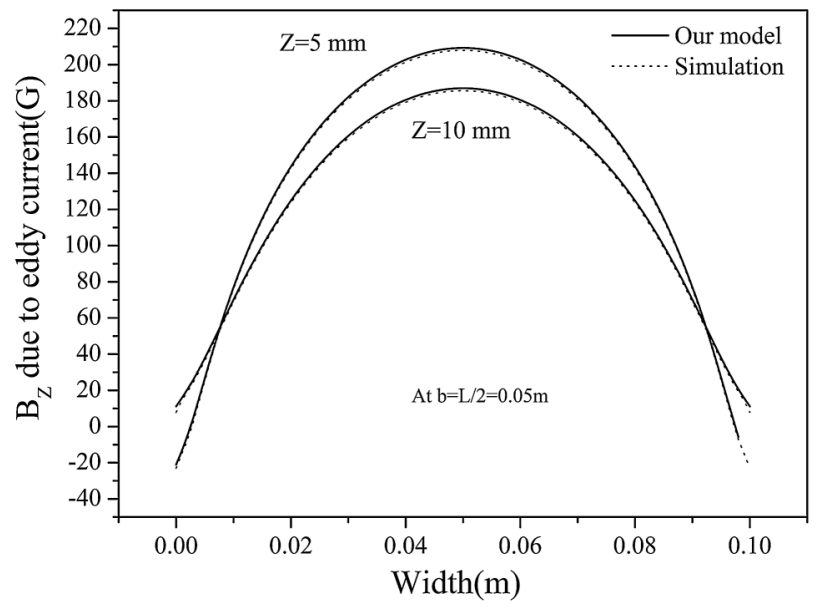

FIG. 8. Vertical component of the magnetic field due to eddy current flow in the SS plate at $50 \mathrm{~Hz}$ frequency and $1.2180 \mathrm{~T}$ peak field for two distances $z=5$ and $10 \mathrm{~mm}$ from the plate, respectively. Simulation results (dotted lines) are also plotted for comparison.

expected one. However, the nature of the field variation is very similar to the actual values.

We will now examine the validity of our model when the plate is exposed to a space dependent time varying field, i.e., case II. The vacuum chambers of a storage ring are placed between the poles of a dipole magnet. A large fraction of the total cross section of the vacuum chambers lies outside the pole gap of the magnet to facilitate the paths for synchrotron radiation. This exposes the chamber to a nonuniform field. To study the effect analytically, we have simplified this problem and expose a metallic plate to a space dependent time varying magnetic field. A SS plate

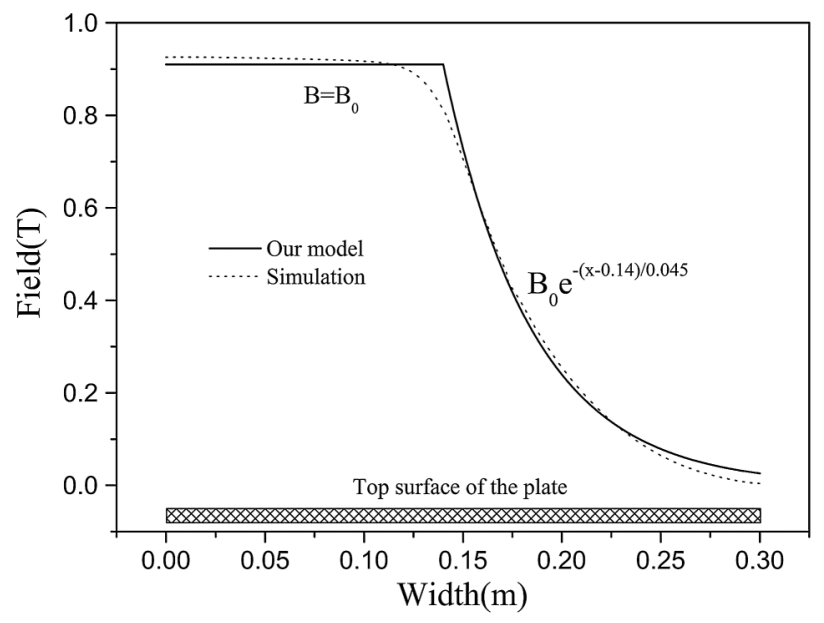

FIG. 9. A SS plate of dimension $(0.3,0.1,0.002) \mathrm{m}^{3}$ is exposed to a space dependent time varying magnetic field. The eddy current generated by such an asymmetric field will be evaluated.

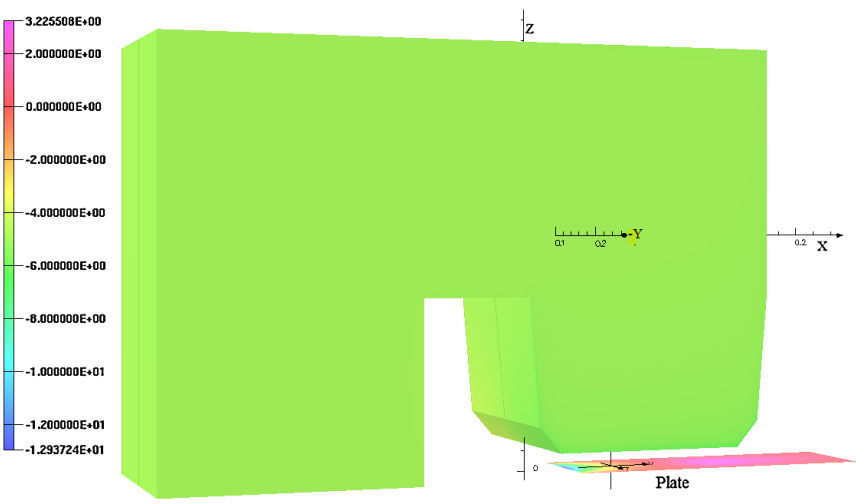

FIG. 10. One half of the 3D model used for simulation study is shown here. Different colors represent the eddy current $\left(\mathrm{A} / \mathrm{mm}^{2}\right)$ in the plate for $50 \mathrm{~Hz}$ frequency. Note that there is no eddy current in the magnet core.

of dimension $(0.3,0.1,0.002) \mathrm{m}^{3}$ is placed in a field which is uniform, $B_{0}$, up to the width $0.14 \mathrm{~m}$ and then the field decreases as $B_{0} \exp \{-(x-0.14) / 0.045\}$, where $B_{0}$ is $0.991 \mathrm{~T}$. To generate such fields for simulation study, a 3D model is developed. The field variation in such a case and the field profile of our model are shown in Fig. 9 and the 3D model is shown in Fig. 10.

The value of the eddy current density in the plate varies from $-12.93724 \mathrm{~A} / \mathrm{mm}^{2}$ (blue color in the Fig. 10) to $3.225508 \mathrm{~A} / \mathrm{mm}^{2}$ (pink color). In the simulation we have assumed the conductivity of the core material of the magnet is zero. Therefore, no eddy current will flow in the core. The color of the magnet core in Fig. 10 represents the material color only.

In some of the storage ring electrons are injected at lower energy and then ramped up to the nominal energy. 


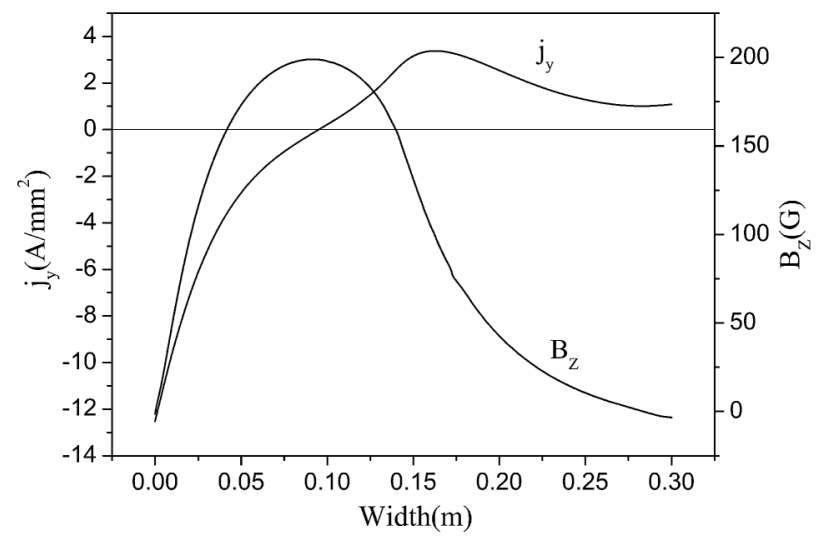

FIG. 11. The $Y$ component of the eddy current density, $j_{y}$ in the SS plate and the field due to the eddy current at $z=10 \mathrm{~mm}$ are plotted for $50 \mathrm{~Hz}$ frequency when the plate is exposed to a nonuniform time varying magnetic field.

Like in Indus 2 at RRCAT, Indore, India, electrons are injected at $0.550 \mathrm{GeV}$ and then the energy is increased to $2.5 \mathrm{GeV}$ [17]. This calculation will be useful to understand the eddy current effect in such storage rings. In case of a full energy injection ring there may be an occasional failure of power supply which is unavoidable in any practical situation [18]. In that case the vacuum chamber will be subjected to a huge force. Prior knowledge of the force helps in proper design of vacuum chambers and its support structure. So, the calculation of force is important here.

The nature of the eddy current and the field produced by the eddy current, when the SS plate is exposed to a time varying field, is calculated using Eq. (17) and plotted in Fig. 11. Here, the eddy current is passing through zero at $0.096 \mathrm{~m}$ and is asymmetric with respect to the midpoint of the uniform field spread, i.e., $0.07 \mathrm{~m}$ from the edge. The magnitude of the eddy current density is more towards the left compared to the right side of the plate. The value of $j_{y}$ is negative for $68.5 \%$ of the plate volume and in the rest of the plate $j_{y}$ is positive but the field is $B_{0}$ up to $0.14 \mathrm{~m}$ and then it reduces exponentially with the increase of width $(X)$. Therefore, the interaction of the eddy current with the applied field generates an unbalanced force on the plate in the negative $X$ direction that has been experimentally observed. The magnetic field produced by the eddy current is also asymmetric. These results are new and have not been reported earlier.

The force acting on the plate can be calculated using Eq. (21). The time variation of the force and the current density is plotted in Fig. 12. The amplitude of the force varies with time. The eddy current increases with time following a sine function whereas the applied field decreases with time following a cosine function. Therefore, the interaction between these two quantities, which gives rise to a force, will be maximum at some intermediate time. This has been nicely depicted by the model as expected.

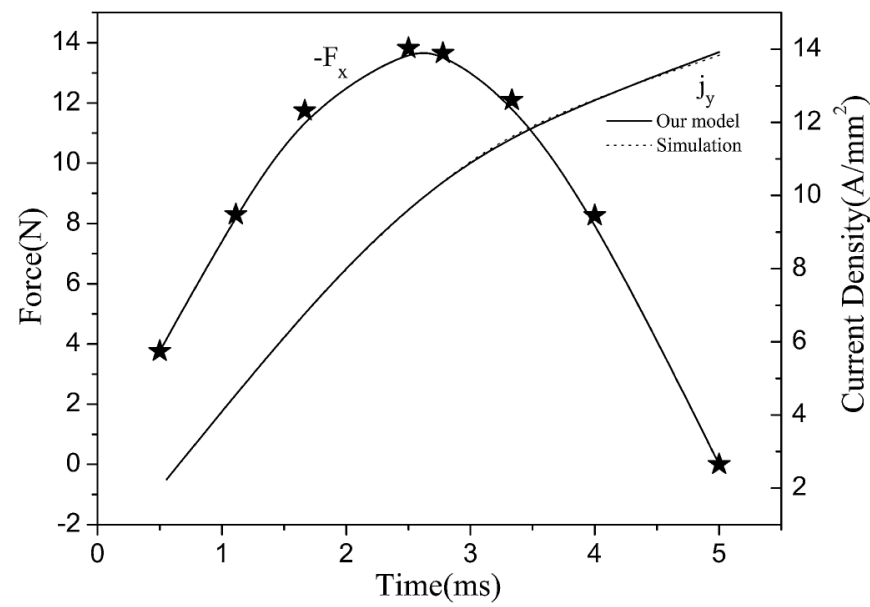

FIG. 12. The time variation of the force and the current density in the SS plate when exposed to the time varying nonuniform field of frequency $50 \mathrm{~Hz}$.

We have also studied the variation of the force on the plate due to the eddy current when exposed to a nonuniform time varying magnetic field for various lengths of the plate keeping its width constant. The frequency and the peak field are taken as $50 \mathrm{~Hz}$ and $0.991 \mathrm{~T}$, respectively. The thickness and the width of the plate are 0.002 and $0.3 \mathrm{~m}$, respectively. The length of the plate is varying from 0.1 to $1.0 \mathrm{~m}$. Our calculation shows that the force on the plate for lengths up to $0.4 \mathrm{~m}$ can be expressed as $F=A+B X+C X^{2}$, where $A, B$, and $C$ are -24.32 , 108.61, and 2600.57, respectively, whereas the force for lengths from 0.4 to $1.0 \mathrm{~m}$ can be expressed as $F=A+$ $B X$, where $A$ and $B$ are -401.28 and 2075.13, respectively. We have plotted the force variation with length in Fig. 13 and the solid line in the plot represents the fitted data using the above equations. The force calculation on the plate may

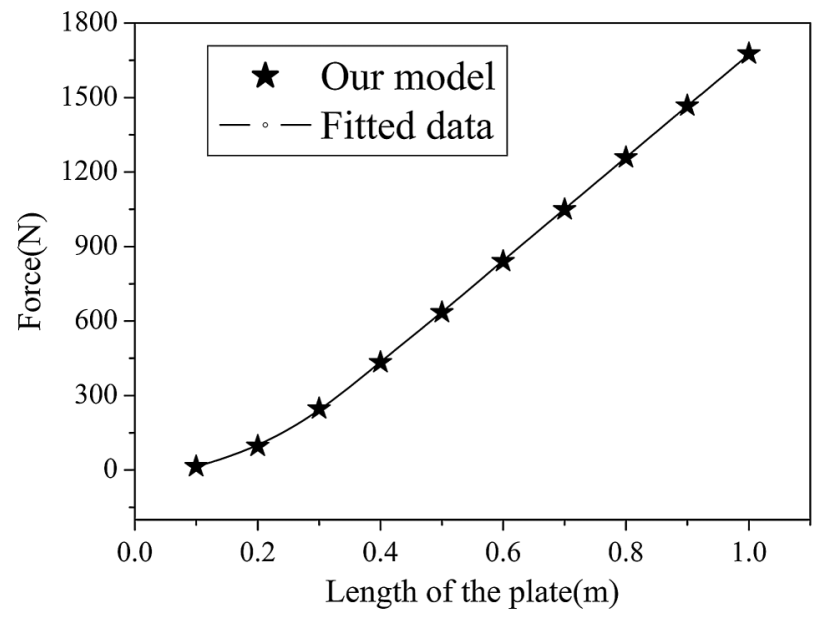

FIG. 13. The variation of the force on the plate due to the eddy current when exposed to a nonuniform time varying magnetic field for various lengths of the plate keeping the width of the plate constant. 
be extended for vacuum chambers of rectangular shape which consists of two such horizontal plates connected by two vertical plates at the end. If we neglect the contributions coming from the vertical plates, then the force will be twice in the case of the chamber.

\section{CONCLUSIONS}

The present model is capable of predicting accurately the eddy current contour of a metallic plate of rectangular shape. We have developed a code in Fortran to calculate various parameters, like eddy current, power loss, field due to eddy current, and force using this model. Our code can provide all the required parameters quickly which helps in understanding the physical behavior of electromagnetic fields better. However, the drawback of the analytical model is that it is difficult to solve if the shape of the plate is complicated. The value of the eddy current predicted by the model is within $0.15 \%$ of the simulation results. It is to be noted that the variation of the eddy current is not linear along the width. Its nature varies at different intermediate time intervals of a cycle. This is one of the new findings of the present work. This model predicts that the nature of the eddy current will be different when the plate is exposed to a uniform and a nonuniform time varying field, i.e., case I and case II of our model. It also predicts the time variation of the eddy current. Power loss predicted by this analytical model for various shapes and frequencies is also in good agreement with the simulation values. Our model predicts the power loss within $0.2 \%$ while earlier analytical models predicted with only a few $\%$ accuracy [3]. This model also predicts that the magnetic field produced by the eddy current will depend on the environment where the plate is kept. The $B$ field produced by it when placed inside the pole gap is calculated. Predictions of our model are accurate because the modification of the $B$ field in the presence of poles is also incorporated in the model. Our results in the case of space varying time dependent field are very interesting. The eddy current distribution in the plate is asymmetric. Because of this nature, the interaction of it with the applied field produces an unbalanced force on the plate. The amplitude of the force varies with time. The eddy current increases with time following a sine function whereas the applied field decreases with time following a cosine function. Therefore, the interaction between these two quantities which gives rise to a force will be maximum at some intermediate time. This has been nicely depicted by the model as expected. The $B$ field produced by the eddy current is also asymmetric. It is expected from this result that when the vacuum chambers, used in storage rings, exposed to such an asymmetric time varying field will produce a deformed $B$ field compared to a symmetric field. We also mentioned that there will be a net force acting on a plate when it is exposed to a nonuniform time varying magnetic field, whereas in the case of a uniform field exposure the resultant force will be zero.

\section{ACKNOWLEDGMENTS}

We gratefully acknowledge Sudhansu S. Mandal for his help in developing the theoretical part of the problem and many stimulating discussions. We also acknowledge Professor A. K. Majumdar and Professor Gurnam Singh for useful discussions and encouragement. We would also like to thank both the referees for many useful comments and suggestions.

[1] A. A. Kim, M. G. Mazarakis, V. I. Manylov, V. A. Vizir, and W. A. Stygar, Phys. Rev. ST Accel. Beams 13, 070401 (2010).

[2] S. Y. Lee, Nucl. Instrum. Methods Phys. Res., Sect. A 300, 151 (1991).

[3] Nikolaos Tsopelas and Nicolaos J. Siakavellas, IEEE Trans. Magn. 44, 4711 (2008); Nicolaos J. Siakavellas, IEEE Trans. Magn. 33, 2245 (1997).

[4] V. Rudnev, D. Loveless, R. Cook, and M. Black, Handbook Of Induction Heating (Marcel Dekker, New York, 2003).

[5] I. Park, I. Kwak, H. Lee, K. Lee, and S. Hahn, IEEE Trans. Magn. 33, 1624 (1997).

[6] Babak Ebrahimi, Mir Behrad Khamesee, and Farid Golnaraghi, J. Phys. D 42, 075001 (2009).

[7] Boris Podobedov, Lynne Ecker, David Harder, and George Rakowsky, in Proceedings of the 23rd Particle Accelerator Conference, Vancouver, Canada, 2009 (IEEE, Piscataway, NJ, 2009), p. 3398.

[8] N. Tsoupas, L. Ahrens, K. A. Brown, J. W. Glenn, and C. J. Gardner, in Proceedings of the 18th Particle Accelerator Conference, New York, 1999 (IEEE, New York, 1999), p. 2918.

[9] Lachaize Antoine, Estimate of Eddy Current Effects in the Vacuum Chamber of the BETA-BEAM RCS (CNRS/ IN2P3/IPNO, Orsay, France, 2007) [http://beta-beam .web.cern.ch/beta-beam/task/docs/RCSeddyCurrents.pdf].

[10] OPERA-3D user guide, Vector Fields Limited, U.K.

[11] Multiphysics simulation software, ANSYS, Inc.

[12] S. H. Kim, APS Report No. LS-291, 2001.

[13] Kuanjun Fan, Izumi Sakai, and Yoshitugu Arakaki, Nucl. Instrum. Methods Phys. Res., Sect. A 597, 142 (2008).

[14] D. A. Gates, J.E. Menard, and R. J. Marsala, Rev. Sci. Instrum. 75, 5090 (2004).

[15] C. Rathjen, in Proceedings of the 8th European Particle Accelerator Conference, Paris, 2002 (EPS-IGA and CERN, Geneva, 2002), p. 2580.

[16] Y. Chung and J. Galayda, APS Report No. LS-200, 1992.

[17] Gautam Sinha and Gurnam Singh, Rev. Sci. Instrum. 79, 123302 (2008).

[18] Gautam Sinha, Subrat Das, S.S. Prabhu, and Gurnam Singh, in Proceedings of the 5th Indian Particle Accelerator Conference, New Delhi, India, 2011. 